NORDITA-94/18 P

hep-th/9404106

April 1994

\title{
Electromagnetic Fields in a Thermal Background
}

\author{
Per Elmfors, ${ }^{1, a}$ and Bo-Sture Skagerstam ${ }^{2}, \mathrm{~b}, \mathrm{c}$ \\ ${ }^{a}$ NORDITA, Blegdamsvej 17, DK-2100 Copenhagen Ø, Denmark \\ ${ }^{b}$ Institute of Theoretical Physics, Chalmers University of Technology \\ and University of Göteborg, S-412 96 Göteborg, Sweden \\ ${ }^{c}$ University of Kalmar, Box 905, S-391 29 Kalmar, Sweden
}

\begin{abstract}
The one-loop effective action for a slowly varying electromagnetic field is computed at finite temperature and density using a real-time formalism. Some earlier errors in the literature are corrected. The effective coupling constant, defined from a purely electric weak-field expansion, behaves at high temperature very differently from the case of a magnetic field, and does not satisfy the renormalization group equation. No thermal corrections to pair production are found at the one-loop level in this formalism.
\end{abstract}

\footnotetext{
${ }^{1}$ Email address: elmfors@nordita.dk.

${ }^{2}$ Email address: tfebss@fy.chalmers.se. Research supported by the Swedish National Research Council under contract no. 8244-311.
} 


\section{INTRODUCTION}

The formulation of quantum field theory in an external field is interesting because of the many applications where the background field is strong [1] and cannot be treated perturbatively. Some relativistic examples are the magnetic field in neutron stars, white dwarfs [2] and heavy-ion collisions [3]. More extreme situations are given by the electroweak phase transition [4] and cosmic strings [5]. Strong (colour) electric fields furthermore appear in some models of hadronization in heavy-ion collisions (for some recent discussions see e.g. $[6,7]$ ). In most of the examples above the fields exist in a thermal heatbath or some non-equilibrium background which is very different from the vacuum.

In this letter the one-loop effective action for a constant electromagnetic field [8] is generalized to finite temperature and chemical potential, $(T, \mu)$. Or, to put it differently, the thermal effective action for a constant magnetic field [9] is generalized to arbitrary constant electromagnetic fields. The case of a pure magnetic field has been treated earlier in $[10,11,9]$ and the incomplete result of [11] was corrected in [9]. The case of a general electromagnetic field has been studied in $[12,13]$ and some corrections to the expressions for the effective action in those papers are presented here. It should be mentioned that the results in the references given above are mutually incompatible. In particular, in this letter no thermal corrections to the pair production rate are found, in agreement with [12] but in disagreement with [13].

For constant external fundamental fields there are various methods of calculating the effective action to all orders in the field, such as a summation of $n$-point functions [14], the method of shifting the field [15] or the tadpole method [16]. Schwinger calculated the effective action for a constant background fieldstrength in QED, for which the gauge fields are non-constant [8]. The proper-time method used in [8] relies on the fact that the solution of the quantum mechanical equations of motion for a particle in the background field can be found explicitly, and an extension to finite $(T, \mu)$ is possible using real-time thermal propagators.

The case of a magnetic field has a rather clear physical interpretation at finite $(T, \mu)$ with the particles in the heatbath occupying the time-independent Landau levels. This situation has been studied in detail in [9]. The physics of a constant electric field at equilibrium is less clear since the field is expected to be screened by the thermal particles. However, since Schwinger's calculation for a general constant electromagnetic field can be extended to finite $(T, \mu)$ in a rather straightforward manner, the question arises about what physical situations it applies to. 
The constant field approximation is satisfied if the relative gradient of the field is smaller than any other scale, i.e. $\left|\partial_{\alpha} F_{\mu \nu}\right| /\left|F_{\mu \nu}\right| \ll\left|e F_{\mu \nu}\right|^{1 / 2}, m_{e}, T$ or $n^{1 / 3}$, where $n$ is the particle density. In this letter we consider only the non-interacting $e^{+} e^{-}$-gas in a time-independent and slowly varying background where the conditions above are satisfied. For instance, the result applies to a shallow potential well with an arbitrary constant magnetic field. A deeper analysis is required to determine whether the result can be used for more general situations. It might, for instance, be possible to have a stationary state with a constant electric field and currents of $e^{+}$and $e^{-}$going in opposite directions. The $e^{+} e^{-}$-annihilation could then act as a friction to keep the particle velocity below the speed of light. Such a state need to be pumped with energy and would not be in equilibrium though stationary. We shall limit the discussion here to the thermal corrections since the vacuum part is easily added when needed.

\section{ONE-LOOP EFFECTIVE ACTION}

The tadpole method [16] is a frequently used method for computing the effective action to all orders in a constant field. It is a relation between the effective action $\Gamma[\phi]$ and the one-point vertex function $\Gamma^{(1)}[\phi ; x]$ for an expansion around a background field $[17]$

$$
\frac{\delta \Gamma[\phi]}{\delta \phi(x)}=\Gamma^{(1)}[\phi ; x]
$$

The situation is similar for QED with a constant background fieldstrength except that the gauge field cannot be constant so it is more difficult to calculate the tadpole in such a background. The tadpole equation at finite temperature and density, to one-loop order, can anyway be rewritten like

$$
\frac{\partial \Gamma[A]}{\partial m}=i \operatorname{Tr}\left\langle x\left|\frac{1}{\pi-m+i \epsilon}-f_{F}\left(p_{0}, A_{0}\right)\left(\frac{1}{\pi-m+i \epsilon}-\frac{1}{\pi-m-i \epsilon}\right)\right| x\right\rangle,
$$

where $\operatorname{Tr}$ is the trace over spin and $x$, and $\pi_{t}=\gamma^{\mu}\left(p_{\mu}-e A_{\mu}\right)$. The thermal part of Eq.(2) is explicitly real as can be seen by taking the complex conjugate and using the cyclicity of the trace.

At this point a few remarks about gauge choice in Eq.(2) are appropriate. Under the gauge transformation $A_{\mu}(x) \mapsto A_{\mu}(x)+\partial_{\mu} \Lambda(x)$, the zero temperature part is transformed to

$$
i \operatorname{Tr}\left\langle x\left|e^{-i e \Lambda(x)} \frac{1}{\pi-m+i \epsilon} e^{i e \Lambda(x)}\right| x\right\rangle,
$$

and thus remains invariant even without using the trace since the Green function is computed at coinciding points. It is equally obvious that the thermal part of Eq.(2) 
is not gauge invariant under time dependent gauge transformations, i.e. when $p_{0}$ and $\Lambda(x)$ do not commute. This gauge dependence originates from the ambiguous division of the system into particles and a background field, where we now only include the particle energy in the Boltzmann factor. The equilibrium state is defined as the state with maximal entropy with a given expectation value of the particle energy

$$
P_{0}=\int d^{3} x \bar{\Psi} \gamma_{0} i \partial_{0} \Psi
$$

and this is a gauge dependent quantity. Moreover, $P_{0}$ in Eq.(4) is not a conserved quantity in a general gauge, in contrast to the total energy of the particles and the field. Only for time independent $A_{\mu}(x)$ is $P_{0}$ separately conserved and that determines the gauge we have to use, namely $\partial_{0} A_{\mu}(x)=0$, up to time independent gauge transformations which, anyway, leave the final result invariant. ${ }^{3}$ To be more precise, $\Lambda(x)$ can a have time dependence of the form $\Lambda(x)=c \cdot t+\lambda(\mathrm{x})$, where $c$ is a constant and $\lambda(\mathrm{x})$ is an arbitrary function. This transformation shifts the potential $A_{0}$ by the constant $c$, and it is necessary to introduce the chemical potential in order to absorb this gauge degree of freedom.

To find thermal one-particle distribution function $f_{F}\left(p_{0}, A_{0}\right)$ we argue that at high temperature it must be reduced to the classical Boltzmann distribution for electrons and positrons, $\exp \left[-\beta\left(\sqrt{\mathbf{p}^{2}+m^{2}} \pm e A_{0} \mp \mu\right)\right]$, and it is the sign of $\left(p_{0}-e A_{0}\right)$ that distinguishes between particles and anti-particles. We therefore write

$$
f_{F}\left(p_{0}, A_{0}\right)=\frac{\theta\left(p_{0}-e A_{0}\right)}{e^{\beta\left(p_{0}-\mu\right)}+1}+\frac{\theta\left(-p_{0}+e A_{0}\right)}{e^{\beta\left(-p_{0}+\mu\right)}+1} .
$$

The distribution function does not have to be chosen to represent an equilibrium distribution. Other choices may be appropriate when the electric field drives the system out of equilibrium.

From Eq.(2) one can follow the calculations in [8] to obtain the thermal part of the effective action. The only difference is that the trace should be taken in the basis $\left|p_{0}, \mathbf{x}\right\rangle$. We find

$$
\begin{aligned}
\frac{\partial \mathcal{L}_{\text {eff }}^{\beta, \mu}(x)}{\partial m^{2}}= & -\frac{1}{2 \pi^{3 / 2}} \int_{-\infty}^{\infty} \frac{d p_{0}}{2 \pi} f_{F}\left(p_{0}, A_{0}\right) \operatorname{Im}\left\{\int_{0}^{\infty} d s e^{2} a b \cot (e s a) \operatorname{coth}(e s b)\right. \\
& \left.\times(i h(s)+\epsilon)^{-1 / 2} \exp \left[-i\left(m^{2}-i \epsilon\right) s+i \frac{\left(p_{0}-e A_{0}\right)^{2}}{h(s)-i \epsilon}\right]\right\}
\end{aligned}
$$

\footnotetext{
${ }^{3}$ Notice that such a gauge was not used in [12].
} 
where

$$
\begin{aligned}
h(s) & =(e F \operatorname{coth} e F s)_{00}, \\
F_{\mu \nu} & =\partial_{\mu} A_{\nu}-\partial_{\nu} A_{\mu} \\
a^{2}-b^{2} & =B^{2}-E^{2} \equiv-\frac{1}{2} \mathcal{F}=\frac{1}{4} F_{\mu \nu} F^{\mu \nu}, \\
a b & =\mathbf{E} \cdot \mathbf{B} \equiv \mathcal{G}, \\
B & =|\mathbf{B}|, \quad E=|\mathbf{E}| .
\end{aligned}
$$

We have added $-i \epsilon$ to $h(s)$ in Eq.(6) in order to get the correct branch. From the $\cot (e s a)$ factor we find that the $s$-integral goes though a number of poles on the real axis, $s=k \pi / e a$, which were not apparent in the original expression. In the case of a pure magnetic field it has been shown [9] that the poles are absent if we only include a finite number of Landau levels. To get the correct result after summing over all Landau levels the $s$-integration contour has to go slightly below the real axis. The expression in Eq.(6) can be directly integrated with respect to $\mathrm{m}^{2}$. The singularity at $s=0$ should be cured by subtracting the $F_{\mu \nu}=0$ part and using a $\zeta$-function regularization as in [9] (or using Eq.(14)).

It is possible to find an explicit expression for $h(s)$ using the following observations. The characteristic equation for the fieldstrength tensor $F \equiv F_{\mu}{ }^{\nu}$ is

$$
\operatorname{det}(\lambda \mathbb{1}-F)=\lambda^{4}-\frac{\mathcal{F}}{2} \lambda^{2}-\mathcal{G}=0,
$$

with the eigenvalues

$$
\lambda= \pm \frac{i}{2}\left(\sqrt{\mathcal{F}^{2}+16 \mathcal{G}^{2}}-\mathcal{F}\right)^{1 / 2}, \quad \pm \frac{1}{2}\left(\sqrt{\mathcal{F}^{2}+16 \mathcal{G}^{2}}+\mathcal{F}\right)^{1 / 2} .
$$

We know that $(F \operatorname{coth} F$ ) has a formal power series expansion involving only even powers of $F$. The Cayley-Hamilton theorem states that $F$ satisfies its own characteristic equation, which can be used to reduce the powers of $F$ down to at most $F^{2}$, so the matrix structure must be

$$
F \operatorname{coth} F=\gamma \mathbb{1}+\delta F^{2} .
$$

Taking the trace and determinant on both sides of Eq.(10) gives two equations to determine $\gamma$ and $\delta$. We find 


$$
\begin{aligned}
h(s)= \\
\quad \frac{e}{4}\left(1-\frac{2\left(E^{2}+B^{2}\right)}{\sqrt{\mathcal{F}^{2}+16 \mathcal{G}^{2}}}\right)\left(\sqrt{\mathcal{F}^{2}+16 \mathcal{G}^{2}}-\mathcal{F}\right)^{1 / 2} \cot \left[\frac{e s}{2}\left(\sqrt{\mathcal{F}^{2}+16 \mathcal{G}^{2}}-\mathcal{F}\right)^{1 / 2}\right] \\
+\frac{e}{4}\left(1+\frac{2\left(E^{2}+B^{2}\right)}{\sqrt{\mathcal{F}^{2}+16 \mathcal{G}^{2}}}\right)\left(\sqrt{\mathcal{F}^{2}+16 \mathcal{G}^{2}}+\mathcal{F}\right)^{1 / 2} \operatorname{coth}\left[\frac{e s}{2}\left(\sqrt{\mathcal{F}^{2}+16 \mathcal{G}^{2}}+\mathcal{F}\right)^{1 / 2}\right] .
\end{aligned}
$$

The relation $s \cdot h(s) \geq 0$ is thus not valid for general electromagnetic field, contrary to the claim in [13]. The quantity $\sum_{\mu=1,2,3}[\ln (\sinh (e F s) / e F s)]_{\mu}^{\mu}$, appearing in the effective action in [12], can be calculated using the same method, and we do not agree on their result either.

The standard way to proceed from Eq.(6) is to deform the s-contour to the imaginary axis. Here, it is not always so useful, except in special cases, since $h(s)$ has zeros on the imaginary axis which give essential singularities from the exponential. On the other hand, the same problem occurs also on the real axis. To be more specific we can consider the following special cases

I.

$$
\mathbf{E} \| \mathbf{B}: h(s)=e E \operatorname{coth} e s E,
$$

II. $\mathbf{E} \perp \mathbf{B}, E>B: h(s)=\frac{e}{E^{2}-B^{2}}\left(E^{2} \sqrt{E^{2}-B^{2}} \operatorname{coth} e s \sqrt{E^{2}-B^{2}}-\frac{B^{2}}{s}\right)$,

III. $\mathbf{E} \perp \mathbf{B}, B>E: h(s)=\frac{e}{B^{2}-E^{2}}\left(\frac{B^{2}}{s}-E^{2} \sqrt{B^{2}-E^{2}} \cot e s \sqrt{B^{2}-E^{2}}\right)$.

In the cases I and II $h(s)$ has no zeros for real $s$ so one kan keep the contour as in Eq.(6). A special case of III is when $E \rightarrow 0$ and we get back the result in [9] by deforming the contour to the imaginary axis. Another interesting special case of $\mathbf{E} \perp \mathbf{B}$ is when $E=B$ in which case we have $a=b=0, h(s)=1 / s$, just like in absence of the external field, but with a potential $A_{0}$. We then find

$$
\begin{aligned}
& \mathcal{L}_{\text {eff }}^{\beta, \mu}\left(F_{\mu \nu}=0, A_{0}\right)=\mathcal{L}_{\text {eff }}^{\beta, \mu}(\mathbf{E} \perp \mathbf{B}, E=B) \\
& =\frac{1}{3 \pi^{2}} \int d p_{0} f_{F}\left(p_{0}, A_{0}\right) \theta\left(\left(p_{0}-e A_{0}\right)^{2}-m^{2}\right)\left(\left(p_{0}-e A_{0}\right)^{2}-m^{2}\right)^{3 / 2},
\end{aligned}
$$

a result similar to the absence of quantum corrections in a propagating plane wave at zero temperature [8]. Notice that, because of the lack of Lorentz invariance, this observation is non-trivial since $\mathcal{L}_{\text {eff }}^{\beta, \mu}$ does not only depend on $\mathcal{F}$ and $\mathcal{G}$. To get Eq.(13) we have used a dimensional regularization as in [9] and the relation

$$
\int_{0}^{\infty} d s s^{\nu} \sin \left(a s-\frac{\pi}{4}\right)=\frac{\Gamma(\nu+1)}{|a|^{\nu+1}}\left\{\begin{aligned}
\sin \frac{\pi}{2}\left(\nu+\frac{1}{2}\right) & \text { if } a>0 \\
-\sin \frac{\pi}{2}\left(\nu+\frac{3}{2}\right) & \text { if } a<0 .
\end{aligned}\right.
$$


In the high temperature limit, and $\mu=0$, we can expand Eq.(13) in powers of $A_{0}$ and extract the Debye screening mass from the coefficient in front of $A_{0}^{2} / 2$. The result, $m_{\gamma}^{2}=e^{2} T^{2} / 3$, agrees with [18] (but not with [13]). Similarly we find the Debye mass at $T=0$ and large $\mu$ to be $m_{\gamma}^{2}=e^{2} \mu^{2} / \pi^{2}$, in agreement with [19].

\section{WEAK E-FIELD EXPANSION}

The $\theta$-function in Eq.(13) arises from the non-analytic behaviour of Eq.(14). If we take $B=0$ and expand the integrand of Eq.(6) in powers of $E$, using Eq.(12), we find that the $s$-integral can be performed term by term using Eq.(14), resulting in the same $\theta$-functions. However, the remaining $p_{0}$-integral becomes infra-red divergent at $\left(p_{0}-e A_{0}\right)^{2}=m^{2}$. This problem occurs already at $\mathcal{O}\left(E^{2}\right)$ in contrast to the case of a $B$-field [9] where the $\mathcal{O}\left(B^{2}\right)$ term can be calculated by a direct expansion of the integrand. It is not a priori clear that a power expansion in $E^{2}$ exists, and from [9] we expect even half-integer powers to occur. It is also plausible that the expansion is only asymptotic just like at zero temperature. Such an expansion would still be useful in many physical situations.

To find the leading weak field expansion we have to be more careful than simply expanding the integrand. Using the notation from Eqs.(12,13), we derive from Eq.(6)

$$
\begin{aligned}
\mathcal{L}_{\mathrm{eff}}^{\beta, \mu}(E)-\mathcal{L}_{\mathrm{eff}}^{\beta, \mu}(0)= & -\frac{1}{4 \pi^{5 / 2}} \int_{-\infty}^{\infty} d \omega f_{F}\left(\omega+e A_{0}\right) \int_{0}^{\infty} \frac{d s}{s^{5 / 2}} \\
& \left\{\left[(\operatorname{sh}(s))^{1 / 2} \cos \left(\omega^{2} s \frac{1-\operatorname{sh}(s)}{s h(s)}\right)-1\right] \cos \left(\left(\omega^{2}-m^{2}\right) s-\frac{\pi}{4}\right)\right. \\
& \left.-(\operatorname{sh}(s))^{1 / 2} \sin \left(\omega^{2} s \frac{1-\operatorname{sh}(s)}{\operatorname{sh}(s)}\right) \sin \left(\left(\omega^{2}-m^{2}\right) s-\frac{\pi}{4}\right)\right\} \\
\equiv & \mathcal{L}_{1}(E)+\mathcal{L}_{2}(E)
\end{aligned}
$$

where $\omega=p_{0}-e A_{0}$. In the first part of the curly bracket in Eq.(15), called $\mathcal{L}_{1}$, we can use the expansion $s h(s) \simeq 1+(s e E)^{2} / 3$ to find the finite $E^{2}$ term

$$
\mathcal{L}_{1}(E)=-\frac{(e E)^{2}}{24 \pi^{2}} \int_{-\infty}^{\infty} \frac{d \omega}{\left(\omega^{2}-m^{2}\right)^{1 / 2}} f_{F}\left(\omega+e A_{0}\right) \theta\left(\omega^{2}-m^{2}\right) .
$$

The infra-red problems arise in when trying to expand $\mathcal{L}_{2}$ in $E$. After introducing a new integration variable $x=\omega^{2}$, and performing a partial integration with respect to $x$, we get

$$
\begin{aligned}
\mathcal{L}_{2}(E)= & \frac{1}{8 \pi^{5 / 2}} \int_{0}^{\infty} d x \int_{0}^{\infty} \frac{d s}{s^{7 / 2}}(s h(s))^{1 / 2} \cos \left(x s-m^{2} s-\frac{\pi}{4}\right) \\
& \frac{d}{d x}\left[\frac{f_{F}\left(\omega+e A_{0}\right)+f_{F}\left(-\omega+e A_{0}\right)}{\omega} \sin \left(x s \frac{1-\operatorname{sh}(s)}{s h(s)}\right)\right] .
\end{aligned}
$$


We can now expand the integrand of $\mathcal{L}_{2}(E)$ to $\mathcal{O}\left(E^{2}\right)$ and find for the total contribution

$$
\begin{aligned}
\mathcal{L}_{\text {eff }}^{\beta, \mu}(E)-\mathcal{L}_{\text {eff }}^{\beta, \mu}(0) & \simeq \frac{(e E)^{2}}{24 \pi^{2}} \int_{m}^{\infty} \frac{d \omega \omega}{\left(\omega^{2}-m^{2}\right)^{1 / 2}} \frac{d}{d \omega}\left(f_{F}\left(\omega+e A_{0}\right)+f_{F}\left(-\omega+e A_{0}\right)\right) \\
& \rightarrow-\frac{(e E)^{2}}{24 \pi^{2}} ; T \rightarrow \infty .
\end{aligned}
$$

In analogy with $[20,9]$ we define an effective fine structure constant by

$$
\frac{1}{\alpha 5_{Q E D}(T)}=\frac{1}{\alpha_{Q E D}}+\left.\frac{1}{\alpha_{Q E D} E} \frac{\partial \mathcal{L}_{\mathrm{eff}}^{\beta, \mu}(E)}{\partial E}\right|_{E \rightarrow 0} .
$$

In the high temperature limit we then have that $\alpha_{Q E D}(T) \rightarrow \alpha_{Q E D} /\left(1-\alpha_{Q E D} / 3 \pi\right)$, showing a completely different behaviour from the $\alpha_{Q E D}$ defined using a magnetic field [9], which satisfies a zero temperature renormalization group equation.

It would, of course, be interesting to go to higher orders in $E$, and also consider a combined $E$ and $B$-field. One will have to perform such an expansion in order to study non-linear effects of wave propagation along the lines of [21].

\section{PAIR PRODUCTION}

One issue that has been discussed in the literature is pair production at finite $T[12,13]$, which we find to be absent from the thermal part of the one-loop contribution. This can be seen directly from Eq.(2) in which the thermal part is manifestly real. Since there is no direct interaction between the particles (no virtual photons) it is not too surprising that the pair production is independent of the thermal state. It depends, of course, on the external field, which is influenced by the thermal background through the equations of motion, but not directly on the thermal particles. On the other hand, one could expect the decay of the vacuum to be suppressed by thermal final-state factors, and a better physical understanding is needed.

It is illustrative to compare with the standard calculation of the effective potential $V(\phi)$ for a spontaneously broken $\lambda \phi^{4}$-model where one may find an imaginary part when the effective mass, $M^{2}(\phi)=\lambda \phi^{2} / 2-m^{2}$, is negative. The expression

$$
\frac{d V(\phi)}{d \phi}=\frac{\lambda \phi}{2} \int \frac{d^{4} p}{(2 \pi)^{4}} f\left(p_{0}\right)\left(\frac{i}{p^{2}-M^{2}+i \epsilon}-\frac{i}{p^{2}-M^{2}-i \epsilon}\right),
$$

is obviously real for any real $M^{2}$. An imaginary part of $V(\phi)$ is found when first calculating Eq.(20) for a positive $M^{2}$ and then performing an (ambiguous) analytic continuation in $M^{2}$. This new function corresponds to, for negative $M^{2}$, a $p_{0}$-integration 
contour which does not follow the real axis but goes above (below) the poles for negative (positive) $p_{0}$, or vice versa. The procedure of obtaining an imaginary part in this way is thus both ambiguous and does not correspond to the $\epsilon$-prescription in Eq.(20). It can, however, be argued from the vacuum calculation [8] that it is the time-ordered prescription that should be used since this uniquely determines the analytical continuation from magnetic to electric fields.

The conclusion we can draw from the example above is that the standard real-time calculation of the one-loop effective potential gives no imaginary part. One should, however, remember that the standard real-time rules are derived under the assumptions of certain factorization properties [22] that may not be fulfilled in presence of unstable modes. In Ref.[7] the pair production was calculated in the imaginary time formalism and was found to be $T$-dependent. It was also pointed out that it was not clear whether their method could be used to compute the decay rate.

\section{Acknowledgement}

We wish to thank D. Persson and P. Liljenberg for close collaboration on related topics that initiated this work. One of the authors (P.E.) have also appreciated the discussions and hospitality of the staff and visitors at ENSLAPP (Annecy), and the financial support from the Swedish Institute.

\section{References}

[1] W. Greiner, B. Müller and J. Rafelski, "Quantum Electrodynamics of Strong Fields", (Springer-Verlag, 1985).

[2] S. T. Shapiro, S. A. Teukolsky, "Black Holes, White Dwarfs, and Neutron Stars, The Physics of Compact Objects" (Wiley, New York, 1983).

[3] R. D. Peccei, J. Solà and C. Wetterich, "New Phase of QED?", Phys. Rev. D37 (1988) 2492.

[4] T. Vachaspati, "Magnetic Fields from Cosmological Phase Transitions", Phys. Lett. B265 (1991) 258.

[5] E. Witten, "Superconducting Strings", Nucl. Phys. B249 (1985) 557.

[6] G. Baur, Multiple Electron-Positron Pair Production in Relativistic Heavy-Ion Collisions: A Strong-Field Effect", Phys. Rev. A42 (1990) 5736. 
[7] A. K. Ganguly, P. K. Kaw and J. C. Parikh, "Thermal Tunneling of q $\bar{q}$ pairs in A-A Collisions", PRL-TH-93/19.

[8] J. Schwinger, "On Gauge Invariance and Vacuum Polarization", Phys. Rev. 82 (1951) 664 and "Particles, Sources and Fields", Vol.3 (Addison-Wesley Pub. Co., 1988).

[9] P. Elmfors, D. Persson and B.-S. Skagerstam, "The QED Effective Action at Finite Temperature and Density", Phys. Rev. Lett. 71 (1993) 480 and "Real-Time Thermal Propagators and the QED Effective Action for an External Magnetic Field", NORDITA-93/78 P and Astropart. Phys. (in press).

[10] W. Dittrich, "Effective Lagrangians at Finite Temperature", Phys. Rev. D19 (1979) 2385.

[11] A. Chodos, K. Everding and D. A. Owen, "QED With a Chemical Potential: The case of a Constant Magnetic Field", Phys. Rev. D42 (1990) 2881.

[12] P. H. Cox, W. S. Hellman and A, Yildiz, "Finite Temperature Corrections to Field Theory: Electron Mass and Magnetic Moment, and Vacuum Energy", Ann. Phys. (N.Y.) 154 (1984) 211.

[13] M. Loewe and J. C. Rojas, "Thermal Effects and the Effective Action of Quantum Electrodynamics, Phys. Rev. D46 (1992) 2689.

[14] S. Coleman and E. Weinberg, "Radiative Corrections as the Origin of Spontaneous Symmetry Breaking" Phys. Rev. D7 (1973) 1888.

[15] R. Jackiw, "Functional Evaluation of the Effective Potential", Phys. Rev. D9 (1974) 1686.

[16] S. Y. Lee and A. M. Sciaccaluga, "Evaluation of Higher-Order Effective Potential With Dimensional Regularization", Nucl. Phys. B96 (1975) 435.

[17] P. Elmfors, K. Enqvist and I. Vilja, "Finite Temperature Effective Action and Thermalization of Perturbations", Nucl. Phys. B (in press).

[18] H. A. Weldon, "Covariant Calculations at Finite Temperature: The Relativistic Plasma”, Phys. Rev. D26 (1982) 1394.

[19] T. Altherr and U. Kraemmer, "Gauge Field Theory Methods for Ultra-Degenerate and Ultra-Relativistic Plasmas", Astropart. Phys. 1 (1992) 133. 
[20] A. Chodos, D. A. Owen and C. M. Sommerfield, "Strong Field Dependence of the Fine Structure Constant", Phys. Lett. B212 (1988) 491.

[21] Z. Bialynicka-Birula and I. Bialynicka-Birula, "Nonlinear Effects in Quantum Electrodynamics. Photon Propagation and Photon Splitting in an External Field", Phys. Rev. D2 (1970) 2341.

[22] A. C. Pearson, "Why the Real-Time Formalism Doesn't Factorise", to be published in Proceedings of the 3rd Workshop on Thermal Field Theories and their Applications, Banff 1993, Eds. F. Khanna, R. Kobes, G. Kunstatter and H. Umezawa, (World Scientific, 1994). 